\title{
Chemical Models of Inner Disks
}

\author{
A. J. Markwick-Kemper \\ University of Virginia, Department of Astronomy, Charlottesville VA 22904, USA \\ email: ajmk@virginia.edu
}

\begin{abstract}
In this paper I describe some of the recent advances made in the modelling of chemistry of protoplanetary disks, and in particular, of the inner regions where $r<100 \mathrm{AU}$. These advances include the treatment of mass-transport processes, the interaction of radiation with chemistry, the augmentation of chemical networks to include isotopic species and their fractionation, and the attempt to connect disk chemistry with solar system bodies like comets. In the spirit of the title of this volume, I also briefly describe what in my opinion are the current challenges facing models of disk chemistry. These include the unification of chemistry, radiation and dynamics, the treatment of gas-grain interaction and the usefulness of observations to discriminate the different models in the literature.
\end{abstract}

Keywords. astrochemistry — comets: general — solar system: formation — stars: planetary systems: formation — stars: planetary systems: protoplanetary disks

\section{Motivation}

Chemistry in protoplanetary disks has been studied by various authors in recent years (for a review, see Markwick \& Charnley 2004a). It is important to study these objects theoretically for reasons related to observations - both current and forthcoming - for example to determine which molecules make good tracers of the disk temperature, density, ionization fraction and radiation field, and to learn how to discriminate between the different models of disk structure currently available. To date, observations have generally been limited to the outer regions of disks, but that is changing. Infrared observations already trace the inner 10 AU, and ALMA will be able to image disks on AU scales, at least in Taurus. Hence the need for good physical/chemical models of the inner disk.

There are other reasons however, for modelling chemistry in disks, which are not so obviously related to their direct observation. We can ask simply what processes affect the chemical structure of the disk - is the coupling between chemistry and dynamics or between chemistry and radiation important? Some recent studies have addressed these questions directly. It turns out, of course, that chemistry, dynamics and radiation are intimately linked in a "triangle of pain", which almost certainly means that no simple back-of-the-envelope calculation is going to be sufficient for accurately describing the chemical composition of disks.

We can realize that, as their name implies, these disks are the precursors of solar systems. We have a great deal of observational data for our solar system, at least in its current state, but also good indications of what it was like primordially from observations of comets and meteorites. There is little argument that the chemical composition of comets is representative of the chemical state of the solar system at some time in its past - although how far back is not so clear cut. Protoplanetary disks form from collapsed interstellar gas and comets form between 5 and $40 \mathrm{AU}$ in the disk. They are perhaps the most pristine objects in solar systems, but are they unprocessed interstellar material? This question is important since it relates directly to what material could have been brought to the early Earth by comets. 


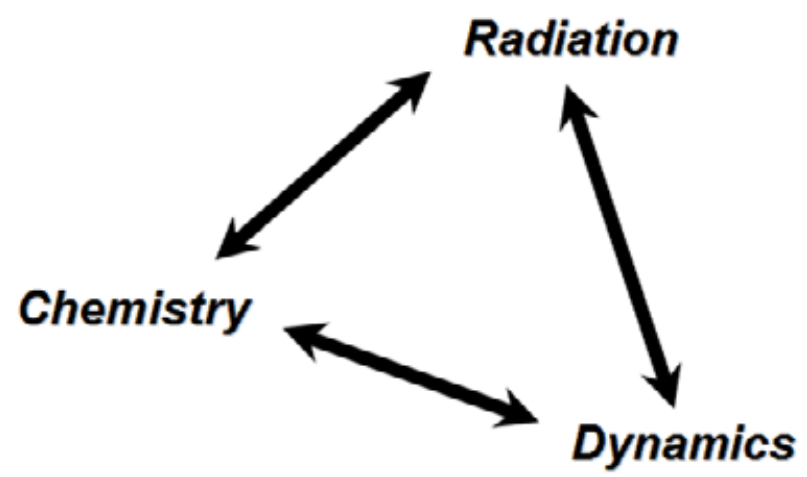

Figure 1. Disk chemistry's "triangle of pain". Chemistry, radiation and dynamics interact, rendering their consistent solution necessary. Unfortunately, this is difficult.

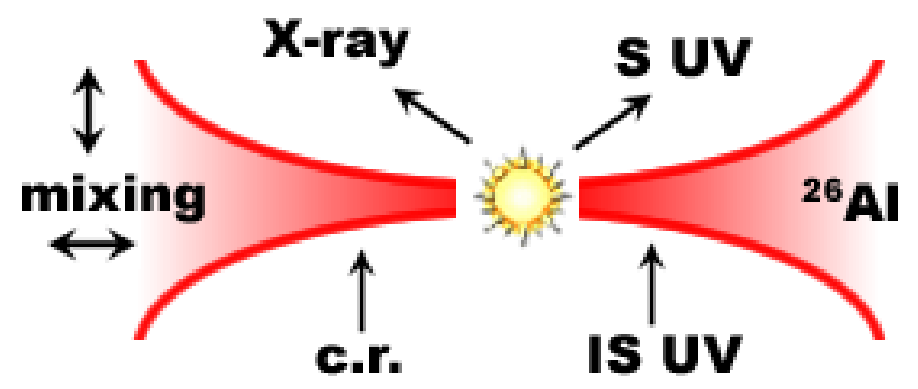

Figure 2. Schematic diagram of the protosolar nebula showing physical processes which affect chemistry. These include sources of ionization (stellar X-rays, stellar UV, interstellar UV, cosmic rays, radionuclides), heating (viscous dissipation, stellar radiation) and transport (e.g., radial mixing, diffusion).

\section{Recent Successes}

Many physical processes should be included in a model of a protoplanetary disk, such as sources of ionization (stellar X-rays, stellar UV, interstellar UV, cosmic rays, radionuclides), heating (viscous dissipation, stellar radiation) and transport (e.g., radial mixing, diffusion; Figure 2). In a model which only considers the midplane, some of these processes can be safely ignored. The temperature and density structure, however, remain crucial. Of the many different models in the literature (see Markwick \& Charnley 2004a), most of them use a different disk structure.

\subsection{Structure}

The temperature profile is important because it controls the return of material into the gas phase from grain surfaces. To illustrate the difference the temperature profile can make on the molecular distributions in the inner disk, Figure 3 compares the distribution of $\mathrm{H}_{2} \mathrm{CO}$ between the models of Markwick et al. (2002) and Millar et al. (2003). The only difference between these two models is that a different physical description of the disk was used in each case. In the former, the hydrostatic disk is heated only by viscous dissipation, while in the latter, the disk is heated both by viscous dissipation and stellar irradiation. The abundance of gas-phase formaldehyde follows the temperature structure of the disk very closely, due to the molecule's thermal desorption from grain surfaces. 

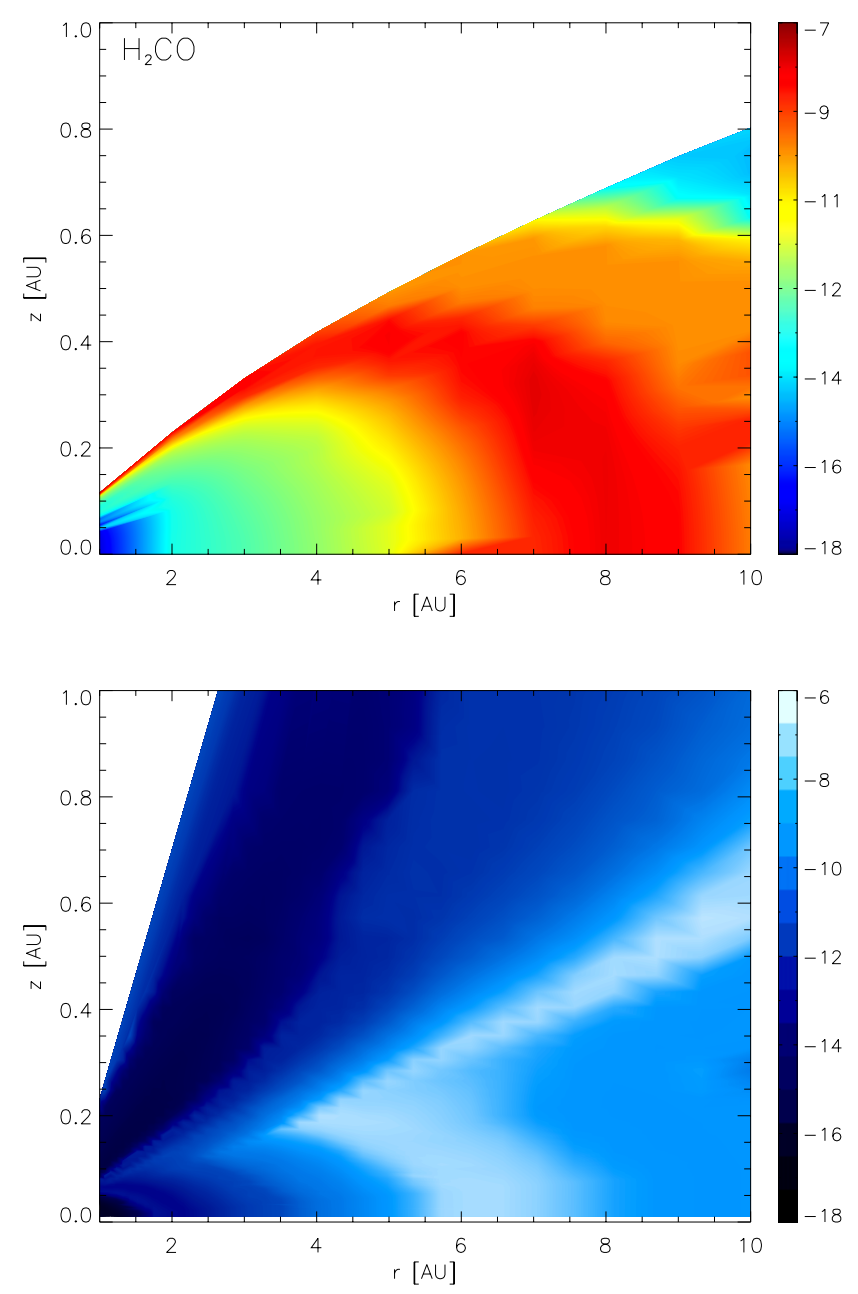

Figure 3. Comparison of the gas-phase $\mathrm{H}_{2} \mathrm{CO}$ distribution in the inner disk produced with models using different physical structures for the disk, but with exactly the same chemistry. Top: Disk heated only by viscous dissipation (Markwick et al. 2002); Bottom: Disk heated by viscous dissipation and stellar radiation (Millar et al. 2003).

\subsection{Transport}

Other works have attempted to identify the differences that including dynamical processes makes on the chemical structure of the disk. Ilgner et al. (2004) compared a model with and without diffusive mass transport. The results were striking, especially for sulphur species like CS (Figure 4), indicating that dynamical processes should certainly be taken into account when modelling chemistry in the inner disk. Semenov et al. (this meeting), presented a 2D mixing model for a disk, in which they found the species $\mathrm{HCN}, \mathrm{HCN}$ and $\mathrm{HCO}^{+}$were most affected by transport processes, and that in fact the $\mathrm{HCN} / \mathrm{HNC}$ line ratio may be a tracer of disk diffusion. Their presentation is available on the conference website at http://asilomar.caltech.edu. 

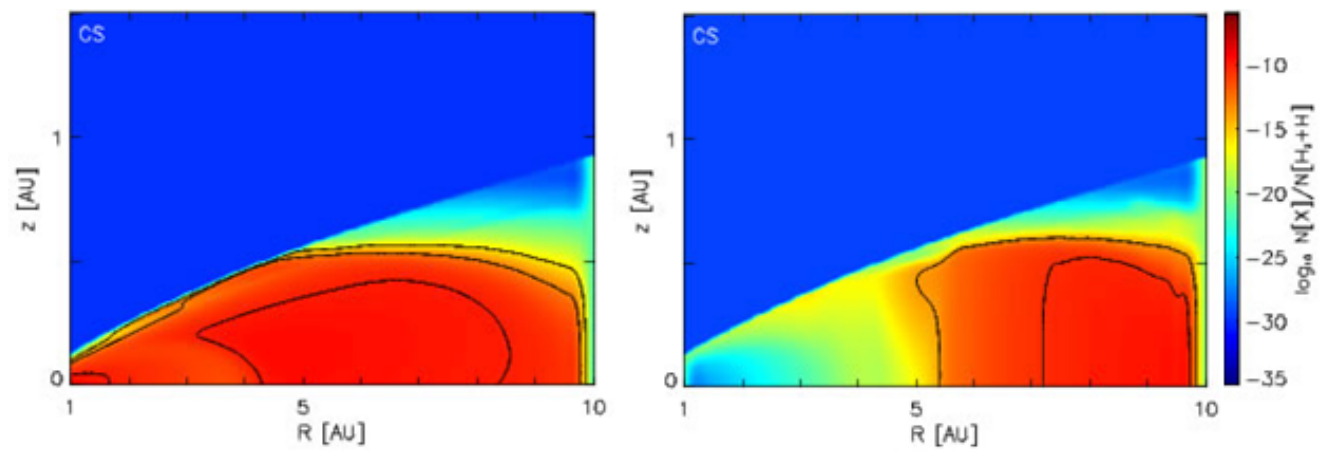

Figure 4. Comparison of the gas-phase CS distribution (log fractional abundance) in the inner disk produced with models with (right) and without (left) diffusive mass transport. (Ilgner et al. 2004).

\subsection{Radiation}

Considering the affect of radiation on chemistry is also important, and van Zadelhoff et al. (2003) used radiative transfer to model UV photo-processes in the disk. They calculated their model with different stellar radiation fields, and found in particular that the $\mathrm{CN} / \mathrm{HCN}$ ratio depends strongly on the stellar spectrum. On the other hand, the $\mathrm{HCO}^{+} / \mathrm{CO}$ ratio does not. Willacy \& Langer (2000) described a detailed photoprocessing calculation. They included photodesorption, finding that significant quantities of molecules can be kept in the gas phase even at visual extinctions in excess of 4 magnitudes.

\subsection{Isotopes}

Aikawa \& Herbst (1999) presented the first disk model to include deuterium fractionation reactions, and therefore were able to draw conclusions between the fractionation in disks and comets. Isotope fractionation consistently proves a valuable tool in astrochemistry, and in the study of disks things are no different. The level of deuterium fractionation in molecules is very sensitive to temperature, and, subject to certain assumptions, can be used to trace the thermal processing history of interstellar material as it passes through the protoplanetary disk stage and becomes incorporated in solar system bodies. One can hope to use carbon and oxygen isotope fractionation in this way too.

To attempt to further investigate the connection between cometary and interstellar material through disks, we constructed a model in which the chemistry is, as usual, based on the UMIST Database for Astrochemistry (http://udfa.net; Le Teuff et al. 2000; Millar et al. 1995), but now augmented to include the deuterium chemistry of Roberts et al. (2003). The initial condition for the chemistry at 100 AU is the output from a dense-core model (using the same chemistry of course), in which the temperature is $10 \mathrm{~K}$ (Markwick-Kemper, unpublished). In these models, the solid $\mathrm{HDO} / \mathrm{H}_{2} \mathrm{O}$ ratio is 0.1 , much higher than the observed ratio in comets. From this initial condition, we calculated two models - one assuming there is no thermal processing of material as it moves inward along the midplane, and another simulating this effect by having the material encounter a region where the temperature rises to $\sim 30 \mathrm{~K}$. These models are described in more detail in Markwick \& Charnley (2004b). Table 1 compares the results so obtained with observations of deuterated molecules in comets. It is clear that the ratios are better matched by the model with thermal processing. The ratios are decreased from their initial values because the heating removes the species from dust grains, the gas-phase 
Table 1. D/H observations and upper limits reported in comets.

\begin{tabular}{lrrr}
\hline Ratio & Observed & Unprocessed & Processed \\
\hline $\mathrm{HDO} / \mathrm{H}_{2} \mathrm{O}$ & 0.0006 & 0.07 & 0.0004 \\
$\mathrm{DCN} / \mathrm{HCN}$ & 0.002 & 0.01 & 0.002 \\
$\mathrm{CH} \mathrm{H}_{3} / \mathrm{CH}_{4}$ & $<0.3$ & 0.1 & 0.14 \\
$\mathrm{HDCO} / \mathrm{H}_{2} \mathrm{CO}$ & $<0.1$ & $>0.06$ & 0.015 \\
$\mathrm{CH}_{2} \mathrm{DOH} / \mathrm{CH}_{3} \mathrm{OH}$ & $<0.04$ & $>0.1$ & 0.0003 \\
$\mathrm{CH}_{3} \mathrm{OD} / \mathrm{CH}_{3} \mathrm{OH}$ & $<0.01$ & $>0.05$ & 0.0004 \\
$\mathrm{C}_{2} \mathrm{HD} / \mathrm{C}_{2} \mathrm{H}_{2}$ & - & 0.003 & 0.09 \\
$\mathrm{DC}$ & - & 0.001 & 0.04 \\
accreting $\mathrm{D} / \mathrm{H}$ & - & 0.6 & 0.005 \\
\hline
\end{tabular}

Notes - D/H observations and upper limits reported in comets, compared with numbers from the two models discussed in the text. Also shown is the accreting gas-phase $\mathrm{D} / \mathrm{H}$ ratio in the two models, and predictions for $\mathrm{C}_{2} \mathrm{HD}$ and $\mathrm{DC}_{3} \mathrm{~N}$. For the unprocessed model, the HDCO and methanol ratios will be lower limits due to grain-surface fractionation.

$\mathrm{D} / \mathrm{H}$ ratios subsequently adjust to the warmer temperature, and then cooling freezes the molecules out onto dust grains at a temperature of around $30 \mathrm{~K}$.

This temperature is supported by other observational results for comets. For example, Kawakita et al. (2004) measured the spin temperatures of ammonia and water molecules in comets. They found that the molecules equilibrated at $26-35 \mathrm{~K}$. In addition, the discovery of crystalline silicates in comet Hale-Bopp indicates that pre-cometary amorphous silicates experienced a significant degree of heating (Wooden 2002).

Table 1 gives predicted values for $\mathrm{C}_{2} \mathrm{HD} / \mathrm{C}_{2} \mathrm{H}_{2}$ and $\mathrm{DC}_{3} \mathrm{~N} / \mathrm{HC}_{3} \mathrm{~N}$. These species were chosen firstly because the main isotopologue has been observed in comets, and secondly because the ratio shows a marked difference between the two models and therefore provides a further observational test. The difference is possible for certain species (like $\mathrm{CH}_{4}$ as well) because there are competing routes to fractionation. At $30 \mathrm{~K}$ for example, the main $\mathrm{H}_{3}^{+}$route is inefficient, but the $\mathrm{C}_{2} \mathrm{H}_{2}^{+}$and $\mathrm{CH}_{3}^{+}$routes, which have different temperature dependencies are not, so species can be fractionated through these ions at higher temperatures.

We now present for the first time results akin to those described above for carbon and oxygen isotope fractionation. The chemistry was augmented again to include ${ }^{13} \mathrm{C}$-, ${ }^{17} \mathrm{O}$ - and ${ }^{18} \mathrm{O}$-bearing species, for carbon chains up to $\mathrm{C}_{3}$. This is a major modification of the chemistry, requiring manifest assumptions about the chemistry of carbon chains, and will be described in detail elsewhere (Markwick-Kemper, in prep.) Table 2 presents results from the same models with and without thermal processing, for comparison with cometary observations. Also presented are some predictions for species for which the main ${ }^{12} \mathrm{C}$ isotopologue has been observed. Of these, CS appears to provide a good test of the model.

\subsection{Fractional Ionization}

Semenov, Wiebe \& Henning (2004) presented a detailed analysis of the source of fractional ionization at various locations in a protoplanetary disk. They successfully used chemical network reduction techniques to isolate the chemistries responsible for providing the ionization, in some cases finding networks with as few as 10 species. Recent work including deuterated species has shown that deuterated forms of $\mathrm{H}_{3}^{+}$will be the dominant ions in the disk midplane (Ceccarelli \& Dominik 2005; Markwick \& Charnley 2004b). Indeed, in the model described above, $\mathrm{D}_{3}^{+}$is by far the most abundant ion in the midplane outside of $40 \mathrm{AU}$. Although the direct detection of $\mathrm{D}_{3}^{+}$in a disk is difficult, because it requires a 
Table 2. ${ }^{12} \mathrm{C} /{ }^{13} \mathrm{C}$ observations reported in comets.

\begin{tabular}{lrrr}
\hline Ratio & Observed & Unprocessed & Processed \\
\hline $\mathrm{CO} / \mathrm{C}^{18} \mathrm{O}$ & & 498.3 & 492.2 \\
$\mathrm{CO} /{ }^{13} \mathrm{CO}$ & & 75.0 & 72.0 \\
${ }^{13} \mathrm{CO} /{ }^{13} \mathrm{C}^{18} \mathrm{O}$ & & 495.7 & 490.7 \\
$\mathrm{CS} /{ }^{13} \mathrm{CS}$ & $95 \pm 12,90 \pm 10$ & 77.9 & 62.2 \\
$\mathrm{CN} /{ }^{13} \mathrm{CN}$ & $93 \pm 10$ & 76.1 & 86.3 \\
$\mathrm{HCN} / \mathrm{H}^{13} \mathrm{CN}$ & $90 \pm 15,109 \pm 22,111 \pm 12$ & 37.6 & 43.1 \\
$\mathrm{C}_{2} / \mathrm{C}^{13} \mathrm{C}$ & $518 \pm 45,470 \pm 40$ & 502.2 & 502.9 \\
$\mathrm{H}_{2} \mathrm{O} / \mathrm{H}_{2}^{18} \mathrm{O}$ & & 80.1 & 73.0 \\
$\mathrm{CO}_{2} /{ }^{13} \mathrm{CO}_{2}$ & & 46.5 & 80.9 \\
$\mathrm{C}_{2} \mathrm{H}_{2} / \mathrm{C}^{13} \mathrm{CH}_{2}$ & & & \\
\hline
\end{tabular}

Notes $-{ }^{12} \mathrm{C} /{ }^{13} \mathrm{C}$ observations reported in comets, compared with numbers from the two models discussed in the text. The input ratios were ${ }^{12} \mathrm{C} /{ }^{13} \mathrm{C}=75, \mathrm{O} /{ }^{18} \mathrm{O}=500$. The terrestrial ratios are ${ }^{12} \mathrm{C} /{ }^{13} \mathrm{C}=89, \mathrm{O} /{ }^{18} \mathrm{O}=498$.

background source against which to see the absorption, this does at least opens up the possibility of using observations of $\mathrm{H}_{2} \mathrm{D}^{+}$and/or $\mathrm{HD}_{2}^{+}$, together with models, to measure the ionization fraction of the midplane, which is of course a parameter of consequence to MHD studies of disks.

\section{Current Challenges}

\subsection{Coupling}

Figure 2 shows some of the processes which ought to be included in a model of the inner disk. However, there are currently no models that include all these processes - no models that survive the "triangle of pain". This is a major current challenge of protoplanetary disk modelling. The models described in the previous section have done well in investigating individually the influence of dynamics and radiation on the chemical structure, and indeed have shown that such interactions are pertinent and should be modelled consistently. What is still required, though, is a true coupling of $2 \mathrm{D}$ radiation hydrodynamics and chemistry.

\subsection{Discrimination}

The many models presented in the literature include different processes and sometimes in different ways. However, it is important to calculate whether or not these differences are actually observable or not. With ALMA, for example, we will be able to image disks on solar system scales, and ideally we would like to be able to discriminate between the various models using these observations. It is not clear that we will be able to. What is really required is that a radiative transfer calculation of the observable line profiles be performed in the same way for each of the different chemical model result sets.

\subsection{Gas-Grain Interaction and Grain-Surface Chemistry}

If the temperature profile is so important to the chemical structure of the inner disk, as model results show, then the binding energies of species to grain surfaces, grainsurface chemical processes, and the exact thermal desorption mechanism are of crucial importance. Unfortunately, it is by no means clear that the values for the binding energies used in models, or the means in which thermal desorption is included, are meaningful. More laboratory work like that described by McCoustra (this volume) is required to 
better characterize gas-grain interactions, and more experiments and calculations like those presented by Watanabe and Charnley, respectively (both this volume), are needed to constrain our expectations of grain-surface chemistry. Finally, all these experimental and theoretical data needs to be incorporated into models.

\subsection{Completeness}

The case of multiple deuterium fractionation in general highlights another of the problems facing astrochemical models. That is, the models cannot make predictions for species which are not included in them. This, above all, is an argument for making the models as chemically complete as possible, and is an argument against the reduction of chemical networks.

\section{Conclusion}

Modelling inner disk chemistry is a complicated affair. There have been many improvements and advances in our understanding of the chemistry occurring in the planet and comet forming region of protoplanetary disks since the last IAU Astrochemistry Symposium in Korea and in this paper I have described some of them. It is my hope that some of the challenges facing these models will be met and we will read about them in the proceedings of the next of these meetings.

\section{References}

Aikawa, Y. \& Herbst, E. 1999, Ap. J. 526, 314

Ceccarelli, C. \& Dominik, C. 2005, A\&SA 440, 583

Ilgner, M., Henning, Th., Markwick, A.J., \& Millar, T.J. 2004, A\&A 415, 643

Kawakita, H., Watanabe, J.-I., Furusho, R., Fuse, T., \& Cremonese, G. 2004, Ap. J. 601, 1152 Le Teuff, Y.H., Millar, T.J., \& Markwick, A.J. 2000, A\&AS 146, 157

Markwick, A.J. \& Charnley, S.B., 2004a, in "Astrobiology: Future Perspectives" ed. P. Ehrenfreund et al., proceedings of ISSI Workshop (Kluwer), in press

Markwick, A.J. \& Charnley, S.B. 2004b, in Formation of Cometary Material, 25th Meeting of the IAU, Joint Discussion 14, proceedings, in press

Markwick, A.J., Ilgner, M., Millar, T.J., \& Henning, Th. 2002, A\& A 381, 632

Millar, T.J., Nomura, H., \& Markwick, A.J. 2003, Astron. Space Sci. 285, 761

Millar, T.J., Farquhar, P.R.A., \& Willacy, K. 1995, A\&AS 121, 139

Roberts, H., Herbst, E., \& Millar, T.J. 2003, Ap. J. 591, L41

Semenov, D.A., Wiebe, D.S., Henning, Th., \& Pavlyuchenkov, Y.N. 2005, IAUS 231 Poster, http://asilomar.caltech.edu

Semenov, D.A., Wiebe, D.S., \& Henning, Th. 2004, A\& $A$ 417, 93

van Zadelhoff, G.-J., Aikawa, Y., Hogerheijde, M.R., \& van Dishoeck, E.F. 2003, A\&SA 397, 789

Willacy, K. \& Langer, W.D. 2000, Ap. J. 544, 903

Wooden, D.H. 2002, Earth, Moon \& Planets 89, 247

\section{Discussion}

MiLlaR: Is grain-surface recombination of $\mathrm{D}_{3}^{+}$included in the model?

MARKWICK-KEMPER: Yes, it is. In fact, all molecular ions in the model are assumed to recombine on grain surfaces.

RAWLINGS: You are right to highlight the importance of knowing the correct values for the binding energies, but desorption is not a binary process. Rather, the temperaturedependence of desorption may be very complex. Does this have significant implications for your models? 
MARKWICK-KEMPER: Yes, the exact details of the desorption process are very important. I referred to desorption as a 'switch' because of the way the temperature changes in the disk. The temperature gradient is so steep that material is thermally desorbed from the grains over a short distance. Of course, the way we build the gas-grain interaction into the models allows this sort of thing to happen. Our model of gas-grain interaction is probably too simplistic.

NEUfELD: Since you have waxed lyrical about the role of theory, I wanted to comment that the fractional ionization in the disk midplane, and the mass of the charge carriers, is a critical parameter in fundamental studies of accretion. Both at your and my institutions, our colleagues have substantial efforts underway to understand the magnetorotational instability; thus the ionization in disks is a crucial point of contact between astrochemistry and the wider astrophysics community.

MARKWICK-KEMPER: I agree. 